\title{
Short communication: Relationship between serum cortisol concentration and defensive behavioral responses of dairy cows exposed to natural infestation by stable fly, Stomoxys calcitrans
}

\author{
I. Vitela-Mendoza, ${ }^{*}$ C. Cruz-Vázquez, ${ }^{* 1}$ J. Solano-Vergara, $†$ and A. Orihuela-Trujillo† \\ *Instituto Tecnológico El Llano Aguascalientes, El Llano, Aguascalientes, 20330 México \\ †Facultad de Ciencias Agropecuarias, Universidad Autónoma del Estado de Morelos, Cuernacava, 62210 México
}

\begin{abstract}
The aim of this study was conducted to evaluate the effect of natural infestation by Stomoxys calcitrans on the behavioral and adrenocortical responses of dairy cattle. Twenty Holstein cows randomly selected were individually sprayed with insecticide once every $7 \mathrm{~d}$, whereas no insecticide was applied to the other 20 animals. The average number of flies per cow was estimated daily, and the frequency of fly-avoidance behaviors was measured daily; plasma cortisol concentration was measured each morning. No flies were ever counted on the treated cows at any time during the experiment, whereas an average of $17.13 \pm 1.14$ ( \pm standard error) flies/d were recorded on untreated cows. Tail movement was the most frequent behavior displayed, with stamps or kicks showing the highest increment rate $(41.2 \times)$ when fly population increased from zero to greater than 51 flies/cow. Cortisol concentration increased to a maximum of $56.81 \pm 39.53 \mathrm{ng} / \mathrm{mL}$ with 26 to 30 flies/ cow per day. Coefficients of determination between the number of flies, cortisol concentration, tail movements, and stamps or kicks were $0.73,0.78$, and 0.81 , respectively. The multiple correlation coefficient was 0.90 , with $81 \%$ of the variation in cortisol concentration explainable by variation in the number of flies per cow and the frequency of fly-avoidance behaviors. It was concluded that plasma cortisol concentration is linearly related to a combination of the number of flies and the frequency of fly-dislodging behaviors, producing a maximum response before reaching maximum fly loads.
\end{abstract} Key words: Stomoxys calcitrans, dairy cattle, fly avoidance behavior, cortisol

\section{Short Communication}

The stable fly, Stomoxys calcitrans (L.), is a cosmopolitan hematophagus pest of cattle. Heavy infestation

Received May 18, 2016.

Accepted August 3, 2016.

${ }^{1}$ Corresponding author: cruva18@yahoo.com.mx is responsible for loss of milk production and weight gain (due to the blood loss caused by adult feeding), annoyance from pain, a possible anaphylactic reaction from fly-derived substances left in the animal, interference with normal eating habits, and increased energy utilization by the animal in its effort to ward off flies (Cruz-Vázquez, 2012; Taylor et al., 2012). Animals may repel or dislodge biting flies by ear twitching, head tossing, leg stamping, muscle flicking, muscle twitching, and tail switching, all with the purpose of chasing flies away and reducing animal discomfort caused by pain from bites and, in general, the stress that ensues. It has also been observed that the fly presence makes the animals reduce the time they remain resting, ruminating, or grazing (Dougherty et al., 1993a, 1994; Mullens et al., 2006). When fly biting intensity is high, the performance of fly-repelling or fly-dislodging behaviors tends to increase (Harris et al., 1987).

In concert with these behavioral responses, a series of physiological changes are expected, with the degree of elicitation dependent on the intensity of exposure to the stressor and the success of the biological responses in coping with the challenge (Moberg, 2000). Measurement of changes in hypothalamic-pituitary-adrenal activity, as indicated by plasma glucocorticoid concentration, is commonly used to demonstrate acute and chronic stress responses in cattle (Minton, 1994). A large body of literature exists indicating the central role of the hypothalamic-pituitary-adrenal axis in adaptation (i.e., effects on energy mobilization), and conversely in failure to adapt (i.e., adverse effects of glucocorticoids on growth, reproduction, and health) to environmental stressors (Zavy et al., 1992; Broom and Johnson, 1993; Tsigos and Chrousos, 2002). Furthermore, little information is available on the relationship between the physiological and behavioral responses of dairy cows to stable fly infestation (Eicher et al., 2001; Mullens et al., 2006). Therefore, the current study examines the relationship between cortisol concentration and defensive behavioral responses of dairy cows exposed to natural stable fly infestation. With this in mind, the first aim of our study was to determine if an intensive insecticide 
treatment would eliminate behavioral and physiological responses in cows. In addition, a second aim was to determine if, under natural infestation, animals showing a high behavioral response will also show a high physiological response.

The study was carried out on a dairy farm located in San Francisco de Los Romo municipality, in the state of Aguascalientes, Mexico. The farm was located at $20^{\circ} 30^{\prime}$ $\mathrm{N}$ and $101^{\circ} 50^{\prime} \mathrm{W}$, which is $2,052 \mathrm{~m}$ above sea level. This region is classified as a semi-arid climate with summer rain; in the region, the stable fly infestation is seasonal, being more intense from the middle of summer to the middle of autumn (Cruz-Vázquez et al., 2007). The farm maintains Holstein cattle in a free confinement system, which is characterized by open pens with dirt floors, shaded areas, and concrete drinking and feeding troughs, providing $40 \mathrm{~m}^{2}$ /animal.

Forty mature Holstein cows were selected; the cows were between 3 and $4 \mathrm{yr}$ old and in their second or third lactation, all having 22 to $25 \mathrm{~d}$ postpartum. Animals were randomly distributed in 2 groups with 20 lactating cows/group. Each group was maintained in a different lodging pen with $800 \mathrm{~m}^{2}$ surface and separated from each other by $50 \mathrm{~m}$. The cows were identified with large numbers $(15 \times 15 \mathrm{~cm})$ painted on their flanks so they could be observed at a distance. All animals were machine milked twice a day (0500 and $1700 \mathrm{~h})$, and a TMR based on corn silage, alfalfa, and grain was offered 4 times daily $(0600,1000,1600$, and $2100 \mathrm{~h}$ ).

The treated group was formed $30 \mathrm{~d}$ before the beginning of the study and was kept in the pen until the end of the experiment, $90 \mathrm{~d}$ later. On the first day of the experiment (July 1), the animals were individually sprayed on their back and legs with insecticide $(7.4 \%$ permethrin) after the morning milking (0500 h). From this point, 8 doses of insecticide were applied to these cows, $7 \mathrm{~d}$ apart until the end of the experiment (August 31 ), regardless of the fly count. No insecticide was applied to the other 20 cows (untreated group).

The Committee on Use and Care of Animals of the Instituto Tecnológico El Llano Aguascalientes approved this project. The owner of the dairy farm gave their consent to study their animals.

The number of stable flies on each of the cows was determined daily during the experimental period (62 d) by direct counting of adult flies found resting or feeding on the front of the legs of the animals, by lateral observation with the aid of binoculars when necessary (Thomas et al., 1989; Cruz-Vázquez et al., 2007). Animals were habituated to the presence of the observer before the experiment as the groups were established. From this date to the end of the experiment, the observer counted the number of flies on each cow in random order by walking calmly among the herd. Flies were counted only on standing animals, and counts were made every $10 \mathrm{~min}$ from 1100 to $1500 \mathrm{~h}$, always by the same person until the end of the study; this person was not aware the experimental status of the animals. These time periods were chosen to avoid feeding management disruptions and because stable flies are most active during the warmer parts of the day (Lysyk, 1995; Gilles et al., 2005). In the infrequent event that a cow was found lying down during a counting episode, she was forced to stand and a count was made during the following 10-min episode.

The individual cow behavior was recorded daily during sixteen 5-min continuous episodes every $15 \mathrm{~min}$ during the same observation periods (Martin and Bateson, 1986; Cockram, 1991) and were recorded by the same person, different from the one carrying out the fly count, also unaware of the experimental status of the animals. Behaviors recorded were (1) stamp or kicks of the front or hind legs, which were recorded as one event each time the cow raised its leg or hit the ground with a foot in a vigorous movement, and (2) tail movement, defined as the travel of the tail from its resting position to one side. If the tail recrossed its resting position, this was recorded as another tail movement (Dougherty et al., 1993a).

Blood samples were collected daily from all cows at $0700 \mathrm{~h}, 1 \mathrm{~h}$ after morning feeding. Cows were walked calmly through an uncovered single-file solid-metalsided straight corridor $(1 \mathrm{~m}$ wide $\times 10 \mathrm{~m}$ long $)$ before the entrance of the milking parlor, where they were individually trapped (not restrained) while the sample was obtained. This procedure was not supposed to affect cortisol levels significantly because they were all tame cows habituated to walk through these facilities before every milking session. Samples $(5 \mathrm{~mL})$ were collected into evacuated glass tubes by venipuncture of the coccigeal vein and were kept cool in ice until the plasma could be separated by centrifugation at 1,000 $\times g$ for $15 \mathrm{~min}$ at environmental temperature $(18 \pm$ $4^{\circ} \mathrm{C}$ ), which occurred within 40 min of collection. The samples were frozen $\left(-20^{\circ} \mathrm{C}\right)$ until analysis. Cortisol concentrations were determined in duplicate by RIA using a commercially available kit (Pantex, Santa Monica, CA), the use of which has been validated by Godfrey et al. (1991). The interassay coefficient of variation was $3.16 \%$.

The average number of flies per cow per day was calculated by dividing the number of flies on each cow among the number of times that the counts were performed on each individual, depending on the number of times a cow was found lying down, as counts were conducted only on standing animals. Using this schedule, the number of flies on each cow was counted a maximum of 25 times/d. 
Behaviors are presented as the average frequency per cow per hour of observation, calculated by summing the frequencies measured in each of the four 5-min observations periods in each hour and taking a mean value over the $4 \mathrm{~h}$ of daily observation. The t-test $(P<0.01)$, for 2 independent samples were used to compare treated versus untreated cows for the following variables: number of flies on the front legs, tail movements, and stamps or kicks (Dretzke, 2005). The Spearman's correlation coefficient $(P<0.05)$ for ranked data $\left(\mathrm{r}_{\mathrm{s}}\right)$ was used to examine correlations between the number of flies, cortisol concentration, and the frequency of behavioral events (Dretzke, 2005). Correlations were made using 1 measure/cow. Individuals under study were ranked in 2 ordered series (behavioral and physiological) and compute under the expression:

$$
\mathrm{r}_{\mathrm{s}}=\frac{\sum x^{2}+\sum y^{2}-\sum d^{2}}{\sqrt{\sum x^{2} \sum y^{2}}}
$$

where $x=$ ranking of the $x$ variable (cortisol concentration), $y=$ ranking of the $y$ variable (frequency of behavioral events), and $d=$ the difference in ranks on the 2 variables. Multiple regression $(P<0.01)$, was used to analyze the effect of variation in the number of flies $\left(\mathrm{X}_{1}\right)$ and in the frequency of fly-dislodging behaviors $\left(\mathrm{X}_{2}\right.$ and $\mathrm{X}_{3}$; where $\mathrm{X}_{2}=$ tail movements/cow per hour, $\mathrm{X}_{3}=$ stamps or kicks/cow per hour) on morning plasma cortisol concentration (criterion variable; $\hat{Y}$ ), according to the model

$$
\hat{\mathrm{Y}}=\beta_{0}+\beta_{1} \mathrm{X}_{1}+\beta_{2} \mathrm{X}_{2}+\beta_{3} \mathrm{X}_{3} \text { (Dretzke, 2005), }
$$

where $\beta_{0}=$ intercept; $\beta_{1}=$ beta weight for the number of flies; $\beta_{2}=$ beta weight for the frequency of tail movements/cow per hour; and $\beta_{3}=$ beta weight for stamps or kicks/cow per hour. The number of stable flies alighting on the front legs of the cows varied from zero in the treated cows to up to 55 flies/cow per day in untreated animals. No flies were ever counted on the treated cows at any time during the experiment, whereas an average of $17.13 \pm 1.14$ flies $/ \mathrm{d}(P<0.01)$ were recorded on untreated cows. The frequency of tail movement was $14.1 \pm 5.5$ and $180.6 \pm 16.1$ events/cow per hour in treated and untreated cows with 51 to 55 flies/cow, respectively. Tail movement was the most frequent behavior observed, even when no stable flies were registered. However, even though stamps or kicks were less frequent, this behavior showed the highest increment rate $(41.2 \times)$ when the frequency was compared between zero ( $2.1 \pm 1.2$ events/cow per hour) to greater than 51 flies/cow $(86.5 \pm 1.4$ events/cow per hour).
The average morning plasma cortisol concentration for treated cows was $2.5 \pm 1.8 \mathrm{ng} / \mathrm{mL}$. In contrast, cows with 26 to 30 flies/d had the largest $(P<0.01)$ average cortisol concentration of $56.81 \pm 39.53 \mathrm{ng} / \mathrm{mL}$. Morning cortisol concentration $(43.34 \pm 3.37 \mathrm{ng} / \mathrm{mL})$ for the highest load of flies (51 to 55) did not differ $(P>0.05)$ from the 26 to 30 flies load. Correlation coefficients between the number of flies, cortisol concentration, tail movements, and stamps or kicks were $0.73,0.78$, and $0.81(P<0.05)$, respectively. The $\mathrm{R}^{2}$ values between cortisol concentration and tail movements and stamps or kicks were 0.71 and 0.75 , respectively $(P<0.05)$, whereas an $\mathrm{R}^{2}=0.90(P<0.05)$ was found between behavioral events (tail movements and stamps or kicks).

The multiple correlation coefficient was $0.90(P<$ 0.05), indicating a strong linear relationship between the 3 independent variables (number of flies, tail movements and stamp or kick per cow per hour) and the dependent variable (morning plasma cortisol concentration). Eighty-one percent of the variation in cortisol concentration could be accounted by variation in the number of flies and the behavioral responses $(\mathrm{SE}=$ 8.63). The variation in $\hat{Y}$ that is explained by its relationship with the 3 predictor variables (regression) was significant (ANOVA: df $=3 / 11 ; F=11.43 ; P=0.002$ ). The regression equation estimated was

$$
\hat{\mathrm{Y}}=6.478+0.079 \mathrm{X}_{1}+0.054 \mathrm{X}_{2}+0.389 \mathrm{X}_{3} .
$$

Stomoxys calcitrans infestation causes different effects in the cattle; in several studies a lineal relationship has been observed between the number of flies and the number of defensive behaviors, so that the higher the amount of flies, the more defensive behaviors are observed and vice versa. A reduction in defensive behaviors implies less stress and more comfort for the animals, as well as a reduction of the negative effects on milk production and daily weight gain (Eicher et al., 2001; Mullens et al., 2006; Taylor et al., 2012).

In the present study, treated animals occasionally kicked or stamped their feet. In contrast, Dougherty et al. (1993a) reported that cattle grazing in enclosures that completely excluded stable flies did not stamp their feet or hold their feet above the soil surface. This suggests that the kicks and stamps of cows with no flies counted on their front legs may have been responses to other factors. The fact that stamps or kicks displayed the greatest fold change in response to fly population growth could be because this response is probably caused by biting trauma, as this is the preferred feeding region of stable fly. In contrast, the highest frequencies observed for tail switching could be due to a more unspecific reaction, as cows could be responding to 
domestic flies or other stimuli uncontrolled under natural infestations (Mullens et al., 2006). The discomfort induced by the flies, together with the pain and annoyance of the bite, apparently affected the behaviors of the cows by causing them to engage in extra activity, in an attempt to protect themselves, conditions that might lead to a positive correlation between physiological and behavioral responses. Schofield and Torr (2002) reported that, from all the stable flies landing on adult cattle, only $27 \%$ take a full meal (mean feeding time $=147 \mathrm{~s}$ ). Most stable flies leave the host before completing their meal, largely due to disturbance by the host's defensive behavior $(24 \%$, mean time $=59 \mathrm{~s})$ or disturbance of other flies (44\%, $71 \mathrm{~s})$.

According to Harvey and Launchbaugh (1982), cattle infested with Haematobia irritans that received treatment with permethrin showed in a $50 \%$ decrease in tail switching (48 and 96 events/h in treated and untreated cows, respectively). In our experiment, analogous results were observed; in contrast, untreated cow frequencies increased from $2.1 \pm 1.2$ to $86.5 \pm 1.4$ and from $14.1 \pm$ 5.5 to $180.6 \pm 16.1$ when fly population increased from zero to greater than 51 flies/cow for stamp or kicks and tail switching, respectively. Similarly, Dougherty et al. (1993b) found that, under controlled conditions, releases of up to 250 stable flies every 15 min resulted in linear increases in tail movements and hind leg movements in beef cattle of up to 57.5 and 1.7 events/min, respectively. In addition, Mullens et al. (2006) found that weekly herd mean frequencies of fly-repelling behaviors were highly dependent on fly numbers.

Estienne et al. (1991) found that heart and respiration rates, rectal temperatures, and circulating cortisol concentrations were similar among groups of steers exposed to $0,10,20$, or 30 stable flies for three 15 -min periods daily for $14 \mathrm{~d}$. These results are not in accord with the findings in our study, perhaps because intermittent exposure to those levels of stable flies does not evoke physiological responses in beef steers. Nevertheless, our results are more in accord with Schwinghammer et al. (1986), who reported that intense and sustained parasitism of beef cattle by stable flies increased blood cortisol concentrations in about $10 \mathrm{ng} / \mathrm{mL}$ when the number of flies per cow increased from zero to 5 . Heart rates, respiration rates, and rectal temperatures were also increased.

According to Warnes and Finlayson (1987) calves that respond vigorously by tail flicks, foot stamps and head-swings suffer less from attack by $S$. calcitrans than their more placid contemporaries. However, in the present experiment, serum cortisol and dislodging behaviors increased as the fly population multiplied, suggesting a growing stress response. Perhaps it is the persistent need for such behavioral responses, rather than actual biting of the flies, which elicits the higher cortisol responses in cows with higher number of flies. The highly significant relationship between the adrenocortical responses of dairy cows and their relative fly burdens and associated behavioral responses supports the notion that fly-dislodging behaviors indicate higher levels of fly-related stress in dairy cattle exposed to natural infestations of $S$. calcitrans.

It was concluded that plasma cortisol concentration is linearly related to a combination of the number of flies and the frequency of fly-dislodging behaviors, producing a maximum response before reaching maximum fly loads. The number of tail twitches and stamp or kicks are signs that can be monitored more easily than counting the flies on the cows to determine when treatment might be required. This management technique cannot only improve animal welfare, but also may benefit milk producers economically by restricting insecticide applications to times when the behaviors of the cows reflect the discomfort induced by stable flies.

\section{ACKNOWLEDGMENTS}

We gratefully acknowledge Ngaio Beausoleil (Institute of Veterinary Animal and Biomedical Sciences, Massey University, Palmerston North, New Zealand) for her careful review of the manuscript and the dairy farm "La Perla," of Aguascalientes, Mexico, for valuable collaboration.

\section{REFERENCES}

Broom, D. M., and K. G. Johnson. 1993. Stress and Animal Welfare. Chapman and Hall, London, UK.

Cockram, M.S. 1991. Resting behaviour of cattle in a slaughter house lairage. Brit. Vet. J. 147:109-119. http://dx.doi.org/10.1016/00071935(91)90100-2.

Cruz-Vázquez, C. 2012. Infestación por moscas. Pages 61-70 in Parasitología Veterinaria Volúmen III. Artrópodos. V. F. Ibarra, C. J. A. Figueroa, and M. T. Quintero. Ed. UNAM, México City, México.

Cruz-Vázquez, C., M. Ramos-Parra, I. Vitela-Mendoza, Z. GarcíaVázquez, and M. T Quintero-Martínez.. 2007. Relationships between stable fly infestation with some physical facility characteristics and sanitation practices in several dairy farms in the state of Aguascalientes, Mexico. Vet. Parasitol. 149:246-250. http:// dx.doi.org/10.1016/j.vetpar.2007.08.020

Dougherty, C. T., F. W. Knapp, P. B. Burrus, D. C. Willis, and N. W. Bradley. 1993b. Face flies (Musca autumnalis De Geer) and the behaviour of grazing beef cattle. Appl. Anim. Behav. Sci. 35:313-326. http://dx.doi.org/10.1016/0168-1591(93)90083-2.

Dougherty, C. T., F. W. Knapp, P. B. Burrus, D. C. Willis, and P. L. Cornelius. 1994. Moderation of grazing behavior of beef cattle by stable flies (Stomoxys calcitrans L.). Appl. Anim. Behav. Sci. 40:113-127. http://dx.doi.org/10.1016/0168-1591(94)90076-0.

Dougherty, C. T., f. W. Knapp, P. B. Burrus, D. C. Willis, P. L. Cornelius, and N. W. Bradley. 1993a. Multiple releases of stable flies (Stomoxys calcitrans L.) and behaviour of grazing beef cattle. Appl. Anim. Behav. Sci. 38:191-212. http://dx.doi.org/10.1016/01681591(93)90019-L.

Dretzke, B. J. 2005. Statistics with Microsoft Excel. Pearson Prentice Hall, Upper Saddle River, NJ. 
Eicher, S. D., J. L. Morrow-Tesch, J. L. Albright, and R. E. Williams 2001. Tail-docking alters fly numbers, fly-avoidance behaviours, and cleanliness, but no physiological measures. J. Dairy Sci. 84:18221828. http://dx.doi.org/10.3168/jds.S0022-0302(01)74621-8.

Estienne, M. J., F. W. Knapp, J. A. Boling, and J. G. Burg. 1991. Physiological and nutritional responses of beef steers exposed to stable flies (Diptera: Muscidae). J. Econ. Entomol. 84:1262-1265. http://dx.doi.org/10.1093/jee/84.4.1262.

Gilles, J., J. F. David, and G. Duvallet. 2005. Effects of temperature on the rate of increase of Stomoxys calcitrans and Stomoxys niger niger (Diptera: Muscidae) from La Reunion Island. J. Med. Entomol. 42:959-965. /http://dx.doi.org/10.1093/jmedent/42.6.959.

Godfrey, R. W., S. D. Smith, M. J. Guthrie, R. L. Stanko, D. A Neuendorff, and R. D. Randel. 1991. Physiological responses of newborn Bos indicus and Bos indicus $\mathrm{X}$ Bos taurus calves after exposure to cold. J. Anim. Sci. 69:258-268.

Harris, J. A., J. E. Hillerton, and S. V. Morant. 1987. Effect on milk production of controlling muscid flies, and reducing fly-avoidance behaviour, by the use of fenvalerate ear tags during the dry period. J. Dairy Res. 54:165-171. /http://dx.doi.org/10.1017/ S0022029900025309.

Harvey, T. L., and J. L. Launchbaugh. 1982. Effect of horn flies on behaviours of cattle. J. Econ. Entomol. 75:25-27. http://dx.doi. org/10.1093/jee/75.1.25.

Lysyk, T. J. 1995. Temperature and population density effects on feeding activity of Stomoxys calcitrans (Diptera: Muscidae) on cattle. J. Med. Entomol. 32:508-514. http://dx.doi.org/10.1093/ jmedent/32.4.508.

Martin, P., and P. Bateson. 1986. Measuring Behaviour. Cambridge University Press, London, UK.

Minton, J. E. 1994. Function of the hypothalamic-pituitary-adrenal axis and the sympathetic nervous system in models of acute stress in domestic animals. J. Anim. Sci. 72:1891-1898. http://dx.doi. org/10.2134/jas1994.7271891x.
Moberg, G. P. 2000. Biological response to stress: Implications for animal welfare. Pages 1-21 in The Biology of Animal Stress: Basic Principles and Implications for Animal Welfare. G. P. Moberg and J. A. Mench, ed. CABI Publishing, New York, NY.

Mullens, B. A., K. S. Lii, Y. Mao, J. A. Meyer, N. G. Peterson, and C. E. Szijj. 2006. Behavioral responses of dairy cattle to the stable fly, Stomoxys calcitrans, in an open field environment. Med. Vet. Entomol. 20:122-137. http://dx.doi.org/10.1111/j.13652915.2006.00608.x.

Schofield, S., and S. J. Torr. 2002. A comparison of the feeding behaviour of tsetse and stable flies. Med. Vet. Entomol. 16:177-185. http://dx.doi.org/10.1046/j.1365-2915.2002.00361.x.

Schwinghammer, K. A., F. W. Knapp, J. A. Boling, and K. K. Schillo. 1986. Physiological and nutritional response of beef steers to infestations of the stable fly. J. Econ. Entomol. 79:1294-1298. http:// dx.doi.org/10.1093/jee/79.5.1294.

Taylor, D. B., R. Moon, and D. Mark. 2012. Economic impact of stable flies (Diptera: Muscidae) on dairy and beef cattle production. J. Med. Entomol. 49:198-209. http://dx.doi.org/10.1603/ME10050.

Thomas, G. D., I. L. Berry, D. R. Berkebile, and S. R. Skoda. 1989 Comparison of three sampling methods for estimating adult stable fly (Diptera: Muscidae) populations. Environ. Entomol. 18:513520. http://dx.doi.org/10.1093/ee/18.3.513.

Tsigos, C., and G. P. Chrousos. 2002. Hypothalamic-pituitary-adrenal axis, neuroendocrine factors and stress. J. Psychosom. Res. 53:865-871. http://dx.doi.org/10.1016/S0022-3999(02)00429-4.

Warnes, M. L., and L. H. Finlayson. 1987. Effect of host behaviour on host preference in Stomoxys calcitrans. Med. Vet. Entomol. 1:5357. http://dx.doi.org/10.1111/j.1365-2915.1987.tb00322.x.

Zavy, M. T., P. E. Juniewics, W. A. Phillips, and D. L. Von Tungeln. 1992. Effects of cortisol responses in beef calves of different genotypes. Am. J. Vet. Res. 53:551-557. 